\title{
TWO ERGODIC THEOREMS FOR CONVEX COMBINATIONS OF COMMUTING ISOMETRIES ${ }^{1}$
}

\author{
S. A. McGRATH
}

\begin{abstract}
Let $(X, \widetilde{\mathcal{F}}, \mu)$ be a measure space. In this paper we obtain $L^{p}$ estimates for the supremum of the Cesàro averages of combinations of commuting isometries of $L^{p}(X, \mathscr{F}, \mu)$. In particular, we show that a convex combination of two invertible commuting isometries of $L^{p}(X, \mathscr{F}, \mu), p$ fixed, $1<p<\infty, p \neq 2$, admits of a dominated estimate with constant $p /(p-1)$. We also show that a convex combination of an arbitrary number of commuting positive invertible isometries of $L^{2}(X, \mathscr{F}, \mu)$ admits of a dominated estimate with constant 2.
\end{abstract}

1. Introduction. Let $(X, \mathscr{F}, \mu)$ be a $\sigma$-finite measure space and let $T$ be a linear operator mapping $L^{p}(X, \mathscr{F}, \mu)$ into $L^{p}(X, \mathscr{F}, \mu), p$ fixed, $1<$ $p<\infty$. If there exists a constant $c>0$ such that

$$
\int \sup _{n}\left|f, \frac{f+T f}{2}, \cdots, \frac{f+T f+\cdots+T^{n-1} f}{n}\right|^{p} d \mu \leqq c^{p} \int|f|^{p} d \mu
$$

for all $f \in L^{p}(X, \mathscr{F}, \mu)$, then we say that $T$ admits of a dominated estimate with constant $c$. If the norm of $T$ does not exceed 1 , then we say that $T$ is a contraction. We say that $T$ is positive if it maps nonnegative functions to nonnegative functions.

The purpose of this paper is to obtain dominated estimates for certain convex combinations of commuting invertible isometries of $L^{p}(X, \mathscr{F}, \mu)$. In [5, pp. 368-371], A. Ionescu Tulcea showed that if $T$ is an invertible isometry of $L^{p}(X, \mathscr{F}, \mu), p \neq 2$, then it admits of a dominated estimate with constant $p /(p-1)$. For the case $p=2$, she showed that a positive invertible isometry admits of a dominated estimate with constant 2 . We extend these results by showing that if $p \neq 2$, a convex combination of two commuting invertible isometries of $L^{p}(X, \mathscr{F}, \mu)$ admits of a dominated estimate with

Received by the editors December 11, 1972.

AMS (MOS) subject classifications (1970). Primary 47A35; Secondary 28A65.

Key words and phrases. Dominated estimates of $L^{p}$ contractions, positive contractions, convex combinations of commuting isometries, positive fixed functions, normal positive contractions, periodic isometries.

${ }^{1}$ This research is from the author's Ph.D. thesis, University of Minnesota, 1971, written under the supervision of Professor R. V. Chacon.

(c) American Mathematical Society 1973 
constant $p /(p-1)$. In the case $p=2$, we show that a convex combination of an arbitrary number of commuting positive invertible isometries admits of a dominated estimate with constant 2 by obtaining this estimate for normal positive contractions. This last result generalizes a result due to E. M. Stein, who showed in [4, Theorem 2, p. 1896] that positive selfadjoint contractions admit of dominated estimates with constant 4 .

2. Preliminary theorems. All estimates are obtained by applying the following results.

LEMMA 2.1. If $T_{n}(n=1,2, \cdots)$ and $T$ are contractions of $L^{p}(X, \mathscr{F}, \mu)$ such that $T_{n}$ converges strongly to $T$, and such that each $T_{n}$ admits of a dominated estimate with constant $c$, then $T$ admits of a dominated estimate with constant $c$.

THEOREM 2.2. Let $\left(X_{i}, \mathscr{F}_{i}, \mu_{i}\right), i=1,2, \cdots, n$, be $\sigma$-finite measure spaces and let $T_{i}$ be a positive contraction of $L^{p}\left(X_{i}, \mathscr{F}_{i}, \mu_{i}\right)$ into $L^{p}\left(X_{i+1}, \mathscr{F}_{i+1}, \mu_{i+1}\right)$ for $i=1,2, \cdots, n-1$. Let $h_{i} \in L^{p}\left(X_{i}, \mathscr{F}_{i}, \mu_{i}\right)$ be a positive function, $i=1,2, \cdots, n$, such that (i) $T_{i} h_{i} \leqq h_{i+1}$, (ii) $T_{i}^{*}\left(h_{i+1}^{p-1}\right) \leqq$ $h_{i}^{p-1}$, for $i=1,2, \cdots, n-1$. Then we have

$$
\begin{aligned}
\sum_{i=1}^{n} \int \sup \left|f_{i}, \frac{f_{i}+T_{i-1} f_{i-1}}{2}, \cdots, \frac{f_{i}+T_{i-1} f_{i-1}+\cdots+T_{i-1} T_{i-2} \cdots T_{1} f_{1}}{i}\right|^{p} d \mu_{i} \\
\leqq\left(\frac{p}{p-1}\right)^{p} \sum_{i=1}^{n} \int\left|f_{i}\right|^{p} d \mu_{i}
\end{aligned}
$$

where $f_{i} \in L^{p}\left(X_{i}, \mathscr{F}_{i}, \mu_{i}\right)$.

A slightly more general statement of this theorem and its proof can be found in [1, Theorem 2.1, p. 612].

Corollary 2.3. Let $(X, \mathscr{F}, \mu)$ be a $\sigma$-finite measure space and let $T$ be a positive contraction of $L^{p}(X, \mathscr{F}, \mu)$. Suppose there exists a positive function $h \in L^{p}(X, \mathscr{F}, \mu)$ such that either (i) $T h=h$, or (ii) $T h \leqq h$ and $T^{*}\left(h^{p-1}\right) \leqq$ $h^{p-1}$. Then $T$ admits of a dominated estimate with constant $p /(p-1)$.

Proof. If $h$ satisfies condition (ii) then the corollary follows from Theorem 2.2 upon setting $\left(X_{i}, \mathscr{F}_{i}, \mu_{i}\right)=(X, \mathscr{F}, \mu), T_{i}=T, h_{i}=h$, and $f_{i}=$ $f$ for $i=1,2, \cdots, n$ and noting that

$$
\int \sup _{n}\left|f, \frac{f+T f}{2}, \cdots, \frac{f+T f+\cdots+T^{n-1} f}{n}\right|^{p} d \mu
$$

equals the limit of the Cesàro sums. It is shown in [2, Lemma 3.1, p. 267] that if $h$ is a positive fixed function, i.e. $h$ satisfies condition (i), then $T^{*}\left(h^{p-1}\right)=h^{p-1}$. Thus if $T h=h$, then $T$ admits of a dominated estimate with constant $p /(p-1)$. Q.E.D. 
3. Main results. We obtain the estimate for normal contractions first.

Theorem 3.1. Let $(X, \mathscr{F}, \mu)$ be a $\sigma$-finite measure space and let $T$ be a positive normal contraction of $L^{2}(X, \mathscr{F}, \mu)$. Then $T$ admits of a dominated estimate with constant 2.

Proof. Suppose first that $\|T\|<1$. Then $\left\|T^{*}\right\|<1$. Let $f$ be any positive function in $L^{2}(X, \mathscr{F}, \mu)$ and let $g=\sum_{k=0}^{\infty} T^{* k}(f)$. Then $g>0, g \in L^{2}(X, \mathscr{F}, \mu)$, and $T^{*} g \leqq g$. Let $h=\sum_{k=0}^{\infty} T^{k} g$. We have $h>0, h \in L^{2}(X, \mathscr{F}, \mu)$, and $T h \leqq h$. Moreover, $T^{*} h \leqq h$ since $T^{*} h=\sum_{k=0}^{\infty} T^{k}\left(T^{*} g\right) \leqq \sum_{k=0}^{\infty} T^{k} g=h$. Thus $T$ admits of a dominated estimate with constant 2 by Corollary 2.3.

Now suppose $\|T\| \leqq 1$. Define $T_{n}=\left(1-2^{-n}\right) T, n=1,2,3, \cdots$. Then each $T_{n}$ admits of a dominated estimate with constant 2 and consequently so does $T$ since $T_{n}$ converges strongly to $T$. Q.E.D.

COROllary 3.2. Let $T_{i}, i=1,2, \cdots$, be positive invertible isometries of $L^{2}(X, \mathscr{F}, \mu)$ which commute, i.e. $T_{i} T_{j}=T_{j} T_{i}$ for all $i, j$. Then if $\alpha_{i} \geqq 0$, $i=1,2, \cdots$ and $\sum_{i=1}^{\infty} \alpha_{i} \leqq 1$, the convex combination $S=\sum_{i=1}^{\infty} \alpha_{i} T_{i}$ admits of a dominated estimate with constant 2 .

Proof. Since $T_{i}^{*}=T_{i}^{-1}$, each $T_{i}$ is normal and each $T_{i}$ commutes with $T_{j}^{*}, j=1,2, \cdots$. Consequently $S_{n}=\sum_{i=1}^{n} \alpha_{i} T_{i}$ is a positive normal contraction which admits of a dominated estimate by Theorem 3.1. Since $S_{n} \rightarrow S$ strongly, $S$ also admits of a dominated estimate with constant 2 by Lemma 2.1. Q.E.D.

We now consider the case of convex combinations of isometries of $L^{p}(X, \mathscr{F}, \mu), p \neq 2$; To avoid technical difficulties we will assume that $(X, \mathscr{F}, \mu)$ is a Lebesgue space although the results are true in general.

LEMMA 3.3. Let $(X, \mathscr{F}, \mu)$ be a Lebesgue space and $A, B$ invertible isometries of $L^{p}(X, \mathscr{F}, \mu), p \neq 2$. Let $0<\alpha<1$ and $\beta=1-\alpha$. Then given $n>0$ and $f \in L^{p}(X, \mathscr{F}, \mu)$, we have

$$
\begin{aligned}
\frac{1}{n} \sum_{i=0}^{n-1} \int \sup \mid A^{i} f, & \frac{A^{i} f+S A\left(A^{i} f\right)}{2}, \cdots, \\
& \left.\frac{A^{i} f+S A\left(A^{i} f\right)+\cdots+S^{n-i-1} A^{n-i-1}\left(A^{i} f\right)}{n-i}\right|^{p} d \mu \\
& \leqq\left(\frac{p}{p-1}\right)^{p} \int|f|^{p} d \mu,
\end{aligned}
$$

where $S=\alpha+\beta B A^{-1}$.

Proof. It is a result essentially due to Banach (see [3, Theorem 3.1, p. 461] for a proof of a generalized version of Banach's theorem) that an invertible isometry $U$ can be represented in the form $U f(x)=f(\tau x) \cdot r(x)$, 
where $\tau$ is an invertible measurable point transformation and $r$ is a measurable function satisfying $|r|^{p}=d(\tau \mu) / d \mu$. Since $B A^{-1}$ is an invertible isometry, it can be written in the form $B A^{-1}(f(x))=f(\tau x) \cdot r(x)$. Defining $U f(x)=f(\tau x)|r(x)|$, we see that $U$ is a positive invertible isometry which dominates $B A^{-1}$ in the sense that for $n=1,2,3, \cdots$

$$
U^{n}(|f(x)|) \geqq\left|\left(B A^{-1}\right)^{n} f(x)\right|
$$

for any $f \in L^{p}(X, \mathscr{F}, \mu)$. Let $\left\{U_{m}\right\}$ be a sequence of positive periodic isometries which converges strongly to $U$. A proof that such a sequence exists can be found in [2, Lemma 4.3, p. 269]. A positive periodic isometry $T$ of period $n$ ( $T$ has period $n$ if $T^{n}=I$ ) has a positive fixed function since if $h>0$ then $h+T h+\cdots+T^{n-1} h$ is a positive fixed function of $T$. Thus each $U_{m}$ has a positive fixed function $g_{m}$ and consequently so does each operator $S_{m}=\alpha+\beta U_{m}$ since $\alpha+\beta=1$. Letting $T_{i}=S_{m}$, for $i=1,2, \cdots$, $n-1$ in Theorem 2.2 , we have

$$
\begin{gathered}
\sum_{i=1}^{n} \int \sup \left|f_{i}, \frac{f_{i}+S_{m} f_{i-1}}{2}, \cdots \frac{f_{i}+S_{m} f_{i-1}+\cdots+S_{m}^{i-1} f_{1}}{i}\right|^{p} d \mu \\
\leqq\left(\frac{p}{p-1}\right)^{p} \sum_{i=1}^{n} \int\left|f_{i}\right|^{p} d \mu,
\end{gathered}
$$

where $f_{i} \in L^{p}(X, \mathscr{F}, \mu), i=1,2, \cdots, n$. Since this inequality holds for each $S_{m}$, it follows that it holds with $D=\alpha+\beta U$ in place of $S_{m}$ since $S_{m}$ converges to $D$. Since $D$ dominates $S$, we have

$$
\begin{gathered}
\sum_{i=1}^{n} \int \sup \left|f_{i} \frac{f_{i}+S f_{i-1}}{2}, \cdots, \frac{f_{i}+S f_{i-1}+\cdots+S^{i-1} f_{1}}{i}\right|^{p} d \mu \\
\leqq\left(\frac{p}{p-1}\right)^{p} \sum_{i=1}^{n} \int\left|f_{i}\right|^{p} d \mu
\end{gathered}
$$

for $f_{i} \in L^{p}(X, \mathscr{F}, \mu), i=1,2, \cdots, n$.

We let $f_{i}=A^{n-i} f, i=1,2, \cdots, n$ in the preceding inequality and reverse the order of summation, getting

$$
\begin{aligned}
\frac{1}{n} \sum_{i=0}^{n-1} \int \sup \mid A^{i} f, & \frac{A^{i} f+S A\left(A^{i} f\right)}{2}, \cdots, \\
& \left.\frac{A^{i} f+S A\left(A^{i} f\right)+\cdots+S^{n-i-1} A^{n-i-1}\left(A^{i} f\right)}{n-i}\right|^{p} d \mu \\
& \leqq\left(\frac{p}{p-1}\right)^{p} \int|f|^{p} d \mu . \quad \text { Q.E.D. }
\end{aligned}
$$


THEOREM 3.4. Let $(X, \mathscr{F}, \mu)$ be a Lebesgue space and $A, B$ commuting invertible isometries of $L^{p}(X, \mathscr{F}, \mu), p \neq 2$. Let $0<\alpha<1$ and $\beta=1-\alpha$. Then $\alpha A+\beta B$ admits of a dominated estimate with constant $p /(p-1)$.

Proof. Defining $S=\alpha+\beta B A^{-1}$ we have by the preceding lemma that

$$
\begin{gathered}
\frac{1}{n} \sum_{i=0}^{n-1} \int \sup \left|A^{i} f, \frac{A^{i} f+S A\left(A^{i} f\right)}{2}, \cdots, \frac{A^{i} f+\cdots+S^{n-i-1} A^{n-i-1}\left(A^{i} f\right)}{n-i}\right|^{p} d \mu \\
\leqq\left(\frac{p}{p-1}\right)^{p} \int|f|^{p} d \mu
\end{gathered}
$$

for any positive integer $n$ and any $f \in L^{p}(X, \mathscr{F}, \mu)$. Since $A$ and $B$ commute we have

$$
\begin{aligned}
\frac{1}{n} \sum_{i=0}^{n-1} \int \sup & \left|A^{i} f, \frac{A^{i} f+C\left(A^{i} f\right)}{2}, \cdots, \frac{A^{i} f+C\left(A^{i} f\right)+\cdots+C^{n-i-1}\left(A^{i} f\right)}{n-i}\right|^{p} d \mu \\
& \leqq\left(\frac{p}{p-1}\right)^{p} \int|f|^{p} d \mu,
\end{aligned}
$$

where $C=\alpha A+\beta B$. Since $A$ is an isometry it can be written in the form $A g(x)=g(\tau x) r(x)$. It thus follows that

$$
\begin{aligned}
\int \sup & \left|A^{i} f, \frac{A^{i} f+C\left(A^{i} f\right)}{2}, \cdots, \frac{A^{i} f+C\left(A^{i} f\right)+\cdots+C^{n-i-1}\left(A^{i} f\right)}{n-i}\right|^{p} d \mu \\
& =\int \sup \left|A^{i} f, \frac{A^{i} f+A^{i}(C f)}{2}, \cdots, \frac{A^{i} f+A^{i}(C f)+\cdots+A^{i}\left(C^{n-i-1} f\right)}{n-i}\right|^{p} d \mu \\
& =\int\left|A^{i}\left(\sup \left|f, \frac{f+C f}{2}, \cdots, \frac{f+C f+\cdots+C^{n-i-1} f}{n-i}\right|\right)\right|^{p} d \mu \\
& =\int \sup \left|f, \frac{f+C f}{2}, \cdots, \frac{f+C f+\cdots+C^{n-i-1} f}{n-i}\right|^{p} d \mu
\end{aligned}
$$

for $i=0,1, \cdots, n-1$. Thus for any $n$, we have

$$
\begin{array}{r}
\frac{1}{n} \sum_{i=0}^{n-1} \int \sup \left|f, \frac{f+C f}{2}, \cdots, \frac{f+C f+\cdots+C^{n-i-1}(f)}{n-i}\right|^{p} d \mu \\
\leqq\left(\frac{p}{p-1}\right)^{p} \int|f|^{p} d \mu \text { for any } f \in L^{p}(X, \mathscr{F}, \mu) .
\end{array}
$$


Since the Cesàro sums do not exceed $(p /(p-1))^{p} \int|f|^{p} d \mu$, it follows that

$$
\begin{gathered}
\int \sup \left|f, \frac{f+C f}{2}, \cdots, \frac{f+C f+\cdots+C^{n-1} f}{n}\right|^{p} d \mu \\
\leqq\left(\frac{p}{p-1}\right)^{p} \int|f|^{p} d \mu \quad \text { Q.E.D. }
\end{gathered}
$$

It has been pointed out by M. A. Ackoglu that for contractions, the existence of a dominated estimate implies the almost everywhere existence of the Cesàro limit.

Hence we have:

ThEOREM 3.5. Let $(X, \mathscr{F}, \mu)$ be a $\sigma$-finite measure space. Then the individual ergodic theorem holds for positive normal contractions of $L^{2}(X, \mathscr{F}, \mu)$ and for convex combinations of 2 invertible commuting isometries of $L^{p}(X, \mathscr{F}, \mu), p \neq 2$.

\section{BIBLIOGRAPHY}

1. R. V. Chacon and S. A. McGrath, Estimates of positive contractions, Pacific J. Math. 30 (1969), 609-620. MR 40 \#4421.

2. R. V. Chacon and J. Olsen, Dominated estimates of positive contractions, Proc. Amer. Math. Soc. 20 (1969), 266-271. MR 40 \#1579.

3. J. Lamperti, On the isometries of certain function spaces, Pacific J. Math. 8 (1958), 459-466. MR 21 \#3764.

4. E. M. Stein, On the maximal ergodic theorem, Proc. Nat. Acad. Sci. U.S.A. 47 (1961), 1894-1897. MR 24 \#A1367.

5. A. Ionescu Tulcea, Ergodic properties of isometries in $L^{p}$ spaces, $1<p<\infty$, Bull. Amer. Math. Soc. 70 (1964), 366-371. MR 34 \#6026.

U.S. Naval Academy, AnNapolis, Maryland 21402 